\section{$\underset{\substack{\text { hommes } \\ \text { \& migrations }}}{ }$}

\section{Hommes \& migrations}

Revue française de référence sur les dynamiques

migratoires

$1311 \mid 2015$

Femmes et migrations

\title{
Femmes et migrations en Italie
}

Représentations identitaires, associationnisme et ressources citoyennes

\section{Anna Elia}

\section{(2) OpenEdition}

12 Journals

Édition électronique

URL : http://journals.openedition.org/hommesmigrations/3237

DOI : 10.4000/hommesmigrations.3237

ISSN : 2262-3353

Éditeur

Musée national de l'histoire de l'immigration

Édition imprimée

Date de publication : 1 juillet 2015

Pagination : 15-21

ISBN : 978-2-919040-32-2

ISSN : $1142-852 X$

Référence électronique

Anna Elia, « Femmes et migrations en Italie », Hommes \& migrations [En ligne], 1311 | 2015, mis en ligne le 01 juillet 2018, consulté le 10 décembre 2020. URL : http://journals.openedition.org/

hommesmigrations/3237 ; DOI : https://doi.org/10.4000/hommesmigrations.3237 


\title{
FEMMES ET MIGRATIONS EN ITALIE REPRÉSENTATIONS IDENTITAIRES, ASSOCIATIONNISME ET RESSOURCES CITOYENNES
}

par ANNA ELIA, professeure des processus migratoires, territoire et politiques (master en science des administrations publiques) au département de sciences politiques et sociales (DiSPeS) de l'université de Calabre.

\author{
L'activité associative des femmes migrantes en Italie recouvre \\ différentes stratégies sociales. Si l'éducation interculturelle \\ et l'accompagnement des primo-arrivantes dans la société \\ italienne demeurent historiquement leurs priorités, \\ une nouvelle génération de femmes migrantes a redessiné \\ les contours politiques de leurs actions. La lutte contre \\ les discriminations sur le marché du travail se conjugue \\ au souci de maintenir les liens avec leur pays d'origine et leur \\ famille. Une manière de dépasser les frontières des territoires \\ et des représentations.
}

En Italie, la présence de femmes issues de la migration s'inscrit dans un processus de désindustrialisation et d'augmentation de la demande dans le secteur des services à la personne $e^{1}$. À côté des dynamiques démographiques et sociales, et des défaillances de la politique publique d'assistance, les politiques de régularisation représentent un élément déterminant dans l'amplification de la présence des femmes migrantes dans le travail domestique et d'assistance aux familles. Toutefois, leur participation sociale et politique active dans différentes communes a contribué à la formation d'une profession, de services et de compétences toujours plus importantes. C'est dans cette perspective que sont étudiés les réseaux entre femmes migrantes qui s'orientent vers une dimension associative. Néanmoins, certaines recherches ont souligné que, lorsque les ressources et les outils normatifs d'aide font défaut, l'émergence d'une dimension associative migrante féminine, marquée par un cadre ethnique et solidariste, peut conduire à des situations conflictuelles et faire obstacle à une possible reconnaissance individuelle dans une dimension collective ${ }^{2}$.

Cet article entend montrer à quel point, dans le cas de l'associationnisme migrant féminin, cette médiation continue entre le communautaire et l'individuel, le privé et le public, offre aux femmes dans 
la migration un espace de création de réseaux ethniques "alternatifs" ouverts à de nouvelles relations de confiance et à de nouvelles formes d'associations dans l'espace public.

Tout en nous attardant sur différentes formes d'associationnisme des femmes migrantes en Italie, nous nous attacherons de manière particulière à l'utilisation du capital social dans les ouvertures aux formes de dialogue interethnique ; aux pratiques et aux stratégies adoptées dans les processus d'interaction avec les sujets sociaux et institutionnels locaux ; aux changements identitaires liés à l'expérience migratoire et aux stratégies de recomposition d'une dimension associative.

Le cadre théorique de référence adopté s'attache aux questions soulevées par les études sur l'analyse des réseaux et accorde une attention particulière aux relations entre la capacité d'action subjective des migrants et les obligations de

La participation des femmes

dans la migration à

la société civile à travers leurs

associations leur permet

d'une certaine manière

de s'émanciper ou, du moins,

de contourner les obstacles

de la logique d'exclusion de la

citoyenneté nationale.

es régions du Nord et du Centre a été possible gâce à la documentation recueillie et aux échanges avec des représentantes du monde associatif féminin, dans le cadre des activités de formation du master Migrations-Politiques, services sociaux et bonnes pratiques du département de sciences politiques et sociales de l'université de Calabre, durant l'année académique 2012-2013.

\section{Les réseaux comme lieux de production de la subjectivité}

Dans l'interprétation des migrations de la période post-fordienne, l'analyse du processus migratoire du point de vue des migrants, et non pas de l'État et de ses structures ${ }^{4}$, a été accueillie au sein de la littérature critique comme constituant une référence fondamentale dans l'orientation de l'analyse des processus migratoires contemporains en introduisant la notion de subjectivité ${ }^{5}$.

Ce type d'approche souligne la nécessité de concentrer son attention sur le migrant en tant que "personne", dans sa "singularité d'individu". Dans ce cadre d'analyse, les communautés immigrées et les réseaux ethniques deviendraient des espaces "ghettos" à l'intérieur desquels la propre individualité et la propre visibilité sociale des migrants seraient emprisonnées à l'intérieur de la dimension collective (relations de réciprocité mutuelle) de la communauté immigrée ${ }^{6}$. La théorie des réseaux a donc tendance à isoler analytiquement ce qui semble être une modalité d'organisation du processus migratoire, des "liens interpersonnels complexes de parenté, d'amitié et de lieu d'origine "," plutôt que comme l'expression de l'autonomie et la subjectivité des migrations dans les demandes de conditions nouvelles d'existence ${ }^{8}$.Dans cette optique, les réseaux renforceraient les dynamiques de ségrégation des migrants en interprétant à l'avance les tendances du marché du travail dans les économies développées post-fordiennes (voir le cas des assistants aides à domicile pour les personnes âgées).

Si les réseaux ethniques servent de filtre pour l'entrée sur le marché du travail, à l'intérieur d'une

\footnotetext{
3. Anna Elia, “Les migrations en Italie. Pratiques d'intégration au cœur dans un modèle d'exclusion politique et sociale”, in Manuel Boucher (dir.), Intervention sociale, ethnicité et lutte contre le racisme en Europe, Montreuil, Aux lieux d'être, 2008, pp. 37-67; "Réfugiés et demandeurs d'asile dans le sud de l'Italie. Parcours de durabilité sociale dans un système d'État social déstructuré", in Manuel Boucher, Mohammed Belqasmi (dir.), L'État social dans tous ses états. Rationalisations, épreuves et réaction de l'intervention sociale, Paris, l'Harmattan, 2014, pp. 255-268. 4. Abdelmalek Sayad, La Double Absence. Des illusions de l'émigré aux souffrances de l'immigré, Paris, Seuil, 1999. 5. Yann Moulier-Boutang, De l'esclavage au salariat. Économie historique du salariat bridé, Paris, PUF, 1998 ; Sandro Mezzadra, Diritto di fuga. Migrazioni, cittadinanza, globalizzazione, Verona, Ombre corte, 2001. 6. Alejandro Portes, "The economic sociology of immigration: a conceptualoverview", in Alejandro Portes (dir.), The Economic Sociology of Immigration. Essay on Networks, Ethnicity, and Entrepreneurship, New York, Russel Sage Foundation, 1995, pp. 248-279. 7. Dooren Massey, “Economic development and international migration in comparative perspective”, in Population and Development Review, $n^{\circ} 14,1988$, p. 396. 8. Sandro Mezzadra, op. cit.
} 
"régulation microsociale spontanée", le passage à un tissu associatif permet aux migrants de se situer à un niveau intermédiaire, d'allier l'intimité des relations d'entraide (celles qui s'expriment à l'intérieur des réseaux ethniques) et la solidarité publique, destinée aux autres : migrants ou pas. La participation des migrantes à la société civile à travers leurs associations leur permet d'une certaine manière de s'émanciper ou, du moins, de contourner les obstacles de la logique d'exclusion de la citoyenneté nationale. Dans l'espace associatif, elles se mobilisent pour revendiquer leurs droits sociaux mais, en même temps, elles élaborent des réponses concrètes par le bas, en termes de services d'assistance, sociaux, éducatifs, agissant de manière autonome avec le peu de ressources à disposition, obtenant ainsi une marge de négociation face aux décideurs publics.

L'expérience associative, telle une membrane située entre l'individu et les institutions, permet à ces deux espaces de la vie sociale de communiquer et d'échanger, les femmes migrantes qui y participent revêtant ainsi un rôle de médiatrice entre le public et le privé ${ }^{10}$. À l'intérieur de cet espace de césure, les femmes racontent le changement qu'elles vivent à travers leur "pluriappartenance" : la complexité aussi bien face aux séparations qu'aux réunifications familiales ; la revendication de la différence ou un besoin de reconnaissance ; la valorisation des compétences; des trajectoires/stratégies d'émancipation particulières.

\section{Des associations interethniques au service de la lutte sociale}

Jacqueline Andall11 souligne la part importante de l'emploi illégal de travailleuses étrangères dans les familles italiennes, les espaces de travail au noir et au gris étant dus en partie à la nécessité de gérer la pension des personnes âgées. L'auteure s'attache aussi aux facteurs d'ordre social qui expliquent l'incapacité pour les pays du sud de l'Europe, et en

Femmes réfugiées et Italienne dans un atelier du verre en Calabre (C) WALter GReco.

particulier pour l'Italie, d'harmoniser les changements de l'état social et les transformations démographiques, tandis que la présence des travailleuses étrangères peut permettre aux femmes italiennes de concilier leur travail et leurs engagements familiaux sans pour autant changer la relation de genre. Les femmes migrantes entendent relever le défi de leur émancipation par rapport aux rôles de "badante" et de "colf12" qui les exposent à une triple discrimination : de genre, de classe et ethnique. Ce processus revêt une importance toute particulière dans les années 1980, au moment où les femmes primomigrantes cap-verdiennes, brésiliennes et surtout philippines créent fréquemment un type de mobilité horizontale par la conquête d'une certaine autonomie à travers une participation active au sein des 
syndicats. Les revendications destinées à lutter contre le confinement des femmes dans l'espace domestique sont construites autour de la légalité du contrat de travail.

Les années 1990, jusqu’au début des années 2000, sont marquées par un essor de l'associationnisme en général, qu'il soit féminin ou non. Cette phase coïncide avec l'adoption des premières lois sur la réglementation de l'immigration en Italie : la loi 943/86, qui prévoit l'accès aux droits collectifs (y compris le droit d'association) ; la loi 40 de 98 (c.d. Testo Unico sur l'immigration), qui reconnaît les fonctions de représentation des organismes associatifs désignés comme des acteurs privilégiés s'agissant de la mise en œuvre d'initiatives pour l'intégration sociale au niveau des municipalités. Au-delà d'une importante fonction de tutelle, l'associationnisme féminin, particulièrement attentif à la sensibilisation et à la mobilisation de l'opinion publique, revêt un caractère d'advocacy ${ }^{13}$ au travers d'activités permettant la défense et la promotion de valeurs et de droits. La majorité des associations nées durant cette période sont de type multi-ethnique et, souvent, des femmes italiennes en font partie. Selon le premier rapport sur l'associationnisme migrant féminin, cette typologie représente un peu moins de la moitié des associations féminines ${ }^{14}$. Dans cette stratégie de désethnisation des réseaux, l'engagement des femmes migrantes dans le travail bénévole entraîne le partage de connaissances, d'informations, de ressources, générant un capital social de réciprocité exploitable également par ceux qui ne font pas partie des réseaux associatifs ${ }^{15}$.

Nous pouvons citer dans cette typologie d'associations le projet Intrecci, dans la région ÉmilieRomagne, qui met en valeur le travail de plus de soixante associations et organisations de femmes

Fatma : "Quand je ne suis pas en consultation, je suis sur mon ordinateur, mon portable ou ma tablette pour m'informer et m'exprimer sur la Tunisie." @ SOPHIE PASQUET - HANS LuCAS.

étrangères et mixtes présentes sur le territoire régional. Dans le Lazio, l'association No.Di.: "I Nostri Diritti” (nos droits), créée par des femmes immigrées de Rome, a pour objectif de faire prendre conscience des droits des femmes en matière d'éducation, de logement, de famille, de tutelle des mineurs, de santé, de travail.

\section{Les structures associatives face à la déstructuration étatique}

Les exemples cités ci-dessus se réfèrent à un tissu associatif dont les nœuds sont des associations qui, bien qu'opérant principalement sur une base locale, agissent et ont une influence sur tout le territoire national. Par contre, dans certaines régions du sud de l'Italie, le tissu associatif, moins important, revêt une dimension locale qui échappe aux plus récentes recherches statistiques ${ }^{16}$. Ces associations

13. Le concept d'advocacy indique un processus destiné à transformer les intérêts de la population ou des citoyens en droits en influençant les mécanismes de prise de décision politique. Dans ce cas spécifique, l'advocacy a le but aussi d'attirer l'attention de l'opinion publique sur un problème spécifique et d'encourager les décideurs politiques à projeter des solutions possibles. 14. Lea Battistoni, Oursana Samia, $1^{\circ}$ Rapporto sull'associazionismo delle donne immigrate in Italia, Venezia, Fondazione Nilde lotti, 19 ottobre 2012. 15. Alessandro Pizzorno, "Perché si paga il benzinaio. Nota per una teoria del capitale sociale", in Stato e Mercato, $n^{\circ}$ 57, 1999. 16. Le rapport de 2012 sur l'associationnisme féminin en Italie fait état de l'existence de 200 associations de femmes issues de l'émigration. Elles sont réparties sur le territoire régional et se concentrent dans les régions du Centre-Nord, en particulier en Émilie-Romagne (61 associations), dans le Piémont (14) et le Latium (28). Voir Lea Battistoni, Oursana Samia, op. cit. 
puisent leur force dans les réseaux de particuliers (liens familiaux, amicaux, relations de voisinage), un capital social dont la finalité n'est pas seulement individualiste mais réside dans la création d'une action collective ${ }^{17}$. Citons l'exemple des associations dans les communes siciliennes situées dans la zone ionienne et dans les provinces calabraises de Reggio de Calabre et de Cosenza, où sont présentes de nombreuses femmes migrantes souvent exploitées comme aides ménagères Dans ces cas, les réseaux de femmes migrantes servent souvent d'antennes territoriales de lutte contre les discriminations.

Des coordinations d'acteurs de la société civile sont apparues au début du 2000, et dans lesquelles les associations migrantes féminines d'origine différente, contribuent à la création de nouveaux services dans un système de welfare ${ }^{18}$ local déstructuré. Nous pouvons citer l'exemple du Centre informations immigrés, qui gère un guichet public de soutien et d'orientation en partenariat avec l'Assessorat à l'immigration de la province de Cosenza en Calabre. Les représentantes des associations de femmes migrantes de nationalités différentes, primo et nouvelles arrivantes, à travers cette pratique de soutien-orientation, font émerger le "welfare invisible $e^{19 "}$ contribuant à rendre transparente la rencontre entre la demande et l'offre dans le domaine des soins. Elles deviennent des actrices principales s'agissant la dénonciation des conditions de travail para-esclavagistes ${ }^{20}$.

Toujours dans le sud de l'Italie, on assiste à l'installation de femmes refugiées. Leur organisation en réseaux et leur adhésion à des formes d'associationnisme féminin sont encore peu étudiées, malgré leur participation active dans certains projets territoriaux d'accueil ou d'intégration. En particulier, l'arrivée de mères seules en 1998 dans des villages situés sur la côte ionienne calabraise a donné naissance à des coopératives sociales composées de femmes italiennes et de réfugiées afin de récupérer d'anciens métiers. L'expérience toujours en cours dévoile toutefois des faiblesses sur le plan de la participation des femmes réfugiées. Elles ne sont admises que de manière provisoire dans un système de protection qui rend les pratiques et les actes formalisés de développement durable transitoires ${ }^{21}$.

\section{Les nouvelles associations féminines migrantes : entre solidarité et demande d'empowerment}

Durant la seconde moitié des années 2000, l'inconstance des réponses apportées par les institutions locales aux instances de participation des femmes migrantes et l'incertitude quant aux financements publics ont eu des conséquences inévitables sur la promotion de nouveaux services. Elles finissent par attribuer un rôle prépondérant au travail bénévole des femmes migrantes, et ce, au sein de pratiques et d'actes formalisés qui
Les femmes migrantes entendent relever le défi de leur émancipation par rapport aux rôles de "badante" et de "colf" qui les exposent à une triple discrimination : de genre, de classe et ethnique. risquent ainsi de devenir transitoires. L'observation des formes d'associationnisme féminin apparues lors de la dernière décennie met en relief les changements d'objectifs qui passent des instances de revendication politique à

17. Fortunata Piselli, "Capitale sociale: un concetto situazionale e dinamico", in Stato e Mercato, n 57, 1999. 18. Le terme "welfare” est utilisé en Italie dans le langage scientifique et politique pour définir l'État-providence. Ce terme désigne une dimension fondamentale des États modernes industrialisés cherchant à réguler les forces du marché pour maintenir la paix sociale en poursuivant un double objectif : celui de la "protection sociale" (assurances contre les risques et les aléas de la vie) et celui de la "justice sociale" (redistribution des richesses). Au regard de transformations politiques, idéologiques, économiques importantes, aujourd'hui, la question de l'État social (welfare state) fait l'objet de vifs débats associés à une question centrale : la mondialisation de l'économie libérale implique-t-elle inévitablement une baisse, voire une déstructuration complète des protections sociales collectives des citoyens au profit de modèles plus individualistes ? Voir "L'État social' dans tous ses états", appel à contribution, Calenda.org. 19. Maurizio Ambrosini, Immigrazione irregolare e welfare invisibile. II lavoro di cura attraverso le frontiere, Bologna, il Mulino, 2013. 20. Anna Elia, "Les migrations en Italie. Pratiques d'intégration au cœur dans un modèle d'exclusion politique et sociale”, op. cit. 21. Anna Elia, "Réfugiés et demandeurs d'asile dans le sud de l'Italie. Parcours de durabilité sociale dans un système d’État social déstructuré”, op. cit. 22. Lea Battistoni, Oursana Samia, op. cit. 
des finalités plus solidaires sur une base ethnique ${ }^{22}$. Il s'agit d'une tendance liée au durcissement des politiques migratoires italiennes ayant fait un choix sécuritaire. Par rapport à la condition spécifique des travailleuses migrantes, en 2002, le gouvernement italien approuve une loi de régularisation dénommée Bossi-Fini (loi n 189 du 30 juillet 2002), qui prévoit un article spécifique sur l'émergence du travail irrégulier des "badanti". Cette mesure semble reconnaitre les fonctions accomplies par ces travailleuses, mais elle établit de fortes limites à la procédure de régularisation, engendrant des processus de ségrégation sociale qui font de la femme migrante la victime d'une identification totale aux fonctions qu'elle exerce. Dans ce

Faire partie d'un groupe

dont les femmes

sont le pivot représente

un moyen de sortir

des relations de genre

reproduites de manière

stricte dans les lieux

de destination cadre toutefois, une nouvelle génération de femmes migrantes apparaît, qui élabore des formes de résistance et qui pose un regard critique sur le passé migratoire "tracé" ou "subi" par les femmes pionnières de la migration en Italie.

Faire partie d'un groupe dont les femmes sont le pivot représente un moyen de sortir des relations de genre reproduites de manière stricte dans les lieux de destination. Les femmes migrantes sont rendues vulnérables du fait de leur statut considéré comme provisoire et conditionnel, donc révocable à tout moment si le motif principal du séjour n'est plus valable, comme dans le cas du mariage. Cette condition place les femmes du Maghreb, arrivées principalement dans les années 1990, en situation d'isolement et de ségrégation sociale :"Nous recevons beaucoup de signalements de violences conjugales mais il n'y a jamais de plaintes déposées... Ces femmes n'ont pas confiance en la loi et ont peur d'être expulsées... Pourquoi ne pas les sortir de chez elles grâce à un permis de séjour uniquement à leur nom ?" (Souad Sbai, présidente de l'Association des femmes marocaines en Italie, $\mathrm{Acmid}^{23}$ ).

\section{Lutter contre la double discrimination dans le monde du travail}

L'expérience associative personnelle des femmes migrantes rencontre inévitablement le monde du travail. Souvent, à travers leur rôle de badanti, de colf et de baby-sitter - même si elles ont les titres et les compétences pour trouver un autre emploi -, elles sont amenées à soutenir les dynamiques dans lesquelles évolue le travail de soins à la personne qu'elles exercent. Néanmoins, les femmes migrantes réussissent à s'opposer au phénomène de double discrimination de "genre" et "ethnique"24, favorisant leur capacité d'auto-affirmation, en d'autres termes, des parcours d'empowerment évoluant dans la sphère publique. La recherche de nouvelles formes d'empowerment révèle un processus de "coscientization" : une prise de conscience critique et un engagement actif en vue du changement. En ce sens, l'empowerment entraîne aussi bien un travail sur soi que le changement des structures de pouvoir existantes ${ }^{25}$. Dans le cas de l'Association des femmes philippines en Italie, cette prise de conscience implique un travail de reconstruction identitaire. Les femmes qui composent cette association donnent naissance à des formes de résistance aux processus intergénérationnels de ségrégation dans le travail domestique : "la troisième génération de colf". Pour trouver une "nouvelle voie pour sortir du travail domestique", il est nécessaire de casser l'image d'une communauté silencieuse et rassurante due à un certain attachement aux valeurs sociales traditionnelles. Dans ce cas, les femmes migrantes se heurtent à la rigidité des relations solidaires "conditionnelles" ${ }^{26 "}$ et 
surtout à l'absence de ressources et à la non-reconnaissance politique des fonctions d'assistance-orientation dont les associations féminines continuent de s'acquitter. La question de la représentation soulevée devant les institutions et les acteurs sociaux a pour objectif premier de mettre en valeur les compétences des femmes issues de la migration ou, au moins, de faciliter les processus d'apprentissage de la langue italienne, tout en accordant une attention particulière aux enfants qui les ont rejointes, vulnérables dans un contexte où tout droit de citoyenneté leur est refusé27. "Notre communauté est perçue comme une communauté organisée, mais nous réussissons difficilement à convaincre nos compatriotes quỉls doivent faire de l'empowerment. L'intégration, ça ne signifie pas être aide ménagère, car de nombreux compatriotes n'ont pas appris la langue. C'est difficile et nous n'en avons pas les moyens. Les immigrés viennent d'abord nous voir et puis vont chez Caritas, mais personne ne veut faire de bénévolat parce que tout le monde doit travailler pour vivre. Après 16 ans, les jeunes vivent loin des mères qui ne connaissent pas leurs enfants. Ils ne parlent pas la langue italienne et, dans ce cas, on crée la deuxième et la troisième génération de colf. Après 30 ans, cette communauté restant la plus silencieuse, les institutions ne se sont pas aperçues de ce phénomène" (Chiarito Basa, coordinatrice de l'Association des femmes philippines en Italie).

Dans le cas de l'Association des femmes roumaines en Italie, créée en 2009, le processus de "coscientiza-

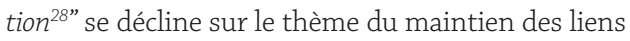
d'appartenance et de la dénonciation des situations dramatiques liées à la double absence des femmes durant la migration. Les mères à l'étranger reproduisent des liens familiaux transnationaux, par exemple par Skype avec leurs enfants. Soutenir la migration d'une compatriote vers l'Italie à travers le partage de la connaissance des lois et de l'expérience contribue à mettre en valeur non seulement l'aspect solidaire de ces actions, mais aussi une nouvelle conscience de ce que représente la diaspora roumaine.

\section{Conclusion}

$\mathrm{Au}$ cours de leur migration en Italie, à l'intérieur de circuits relationnels intra-ethniques ou interethniques, les femmes construisent de nouveaux services au travers deleurs associations et délimitent des espaces d'agency ${ }^{29}$ dans des contextes de dérégulation du marché du travail et du système de welfare local. Elles accumulent de solides expériences dans le travail bénévole et l'éducation interculturelle. En particulier, l'associationnisme multiethnique devient une stratégie politique permettant de dépasser les frontières des réseaux ethniques, offrant des exemples de citoyenneté interculturelle. Durant la dernière décennie, la renaissance de formes d'associationnisme ethnique n'est plus uniquement une forme de repli face à une intégration manquée, mais devient le reflet des changements identitaires liés à l'expérience migratoire de la nouvelle génération de femmes. À travers l'associationnisme, les femmes dans la migration redéfinissent leur appartenance à la communauté, mais surtout elles lui confient la construction de parcours d'empowerment dans lesquels des liens d'appartenance familiale et communautaire entrent en jeu. L'article souligne en particulier la manière dont elles réussissent à rassembler les pans d'une vie marquée par la "double absence ${ }^{30 "}$, en alimentant le lien entre "ici" et "ailleurs" dans des contextes marqués par la déspatialisation physique et symbolique. En ce sens, un aperçu de l'impact de l'émigration sur les lieux d'origine est fondamental, tout comme l'intérêt pour la dimension familiale totalement méconnue dans les politiques de régulation des flux. 